\title{
Mathematical modelling of the degradation behaviour of biodegradable metals
}

\author{
P. Bajger · J. M.A. Ashbourn · V. Manhas · Y. Guyot · K. Lietaert · L. Geris
}

Received: date / Accepted: date

\begin{abstract}
A mathematical model for the biodegradation of magnesium is developed in this study to inspect the corrosion behaviour of biodegradable implants. The aim of this study is to provide a suitable framework for the assessment of the corrosion rate of magnesium which includes the process of formation/dissolution of the protective film. The model is intended to aid the design of implants with suitable geometries. The level-set method is used to follow the changing geometry of the implants during the corrosion process. A system of partial differential equations is formulated based on the physical and chemical processes that occur at the implant-medium boundary in order to simulate the effect of the formation of a protective film on the degradation rate. The experimental data from the literature on the corrosion of a high purity magnesium sample immersed in simulated body fluid is used to calibrate the model. The model is then used to predict the degradation behaviour of a porous orthopaedic implant. The model successfully reproduces the precipitation of the corrosion products on the magnesium surface and the effect on the degradation rate. It can be used to simulate the implant degradation and the formation of the
\end{abstract}

\section{P. Bajger}

Christ Church, University of Oxford, Oxford OX1 1DP, UK

E-mail: p.bajger@uw.edu.pl

J.M.A. Ashbourn

Department of Engineering Science, University of Oxford, Oxford OX1 3PJ, UK

V. Manhas $\cdot$ Y. Guyot $\cdot$ L. Geris

Biomechanics Research Unit, University of Liège, Liège, Belgium

V. Manhas $\cdot$ Y. Guyot $\cdot$ L. Geris

Prometheus, R\&D Division of Skeletal Tissue Engineering, KU Leuven, Leuven, Belgium

V. Manhas · L. Geris

Biomechanics Section, KU Leuven, Leuven, Belgium

K. Lietaert

Department of Materials Engineering, KU Leuven, Leuven, Belgium corrosion products on the surface of biodegradable magnesium implants with complex geometries.

Keywords mathematical modelling · biodegradable metals $\cdot$ level-set method $\cdot$ finite element method

\section{Introduction}

The rate and quality of bone fracture healing depend greatly on the mechanical stability achieved at the fracture site. Nails, pins and plates are often used to stabilise the fracture. Titanium and stainless steel are the most commonly used materials (?). Porous scaffolds are also used in order to provide an initial template for tissue attachment (Guyot et al. 2014). One of the major disadvantages of these fixation devices is the need for a second surgery to remove the implant. Much attention is therefore paid towards the development of biodegradable materials for orthopaedic applications.

Magnesium and its alloys have the potential to be used as a material in the production of biodegradable implants for bone fracture fixation for two reasons. Firstly, magnesium alloys have a density of $1.7-2.0 \mathrm{~g} / \mathrm{cm}^{3}$ and a Young's modulus of $31-45 \mathrm{GPa}$, which are close to those of natural bone $\left(1.8-2.1 \mathrm{~g} / \mathrm{cm}^{3}\right.$ and 3-20 GPa respectively) (Staiger et al., 2006, ?; Xin et al. 2011). This minimises the "stressshielding" effect which is the decrease in bone density following the decrease in loading on the bone as the fixation device takes most of the load (?). Secondly, magnesium is one of the most abundant elements present in human blood. The daily intake of magnesium for a normal adult is about $300-400 \mathrm{mg}$ and excess $\mathrm{Mg}^{2+}$ ions can be gradually absorbed, consumed or excreted by the human body (Song 2007). It has also been reported that the release of $M g^{2+}$ ions by the magnesium-based implants can stimulate hard callus development at fracture sites (Xin et al., 2011). 
One of the concerns about magnesium-based implants is their high degradation rate, particularly in chloride-ioncontaining solutions, such as body fluid. (Song, 2007). In the case of orthopaedic implants, it is important to maintain a low degradation rate for the following reasons: (i) the scaffold must maintain its mechanical integrity long enough for the bone to sufficiently regenerate (Mueller et al. 2009) - it is suggested in (Staiger et al., 2006) that the typical timescale is 12-18 weeks, (ii) magnesium degradation in an aqueous environment is known to produce hydrogen gas which may form harmful bubbles inside the body.

Common methods used to control the biodegradation rate of magnesium $(\mathrm{Mg})$, while at the same time influencing its mechanical properties, are alloying and purification. The degradation behaviour of $M g$-based alloys is affected significantly by the distribution of the intermetallic phases and the presence of impurities ( $\mathrm{Fe}, \mathrm{Ni}, \mathrm{Cu}$, and $\mathrm{Co}$ ) (Wen et al. 2009).

$\mathrm{Fe}, \mathrm{Ni}, \mathrm{Cu}$ and $\mathrm{Co}$ are termed impurities because their presence in the sample may greatly accelerate the degradation rate. As a result of their low solid-solubility limits in $M g$, they tend to form second phases at a very low concentration. These second phases have a low hydrogen overvoltage and act as cathodes for the galvanic corrosion. The degradation rate may therefore increase 10- to 100-fold if the impurity contents are above certain thresholds (Song and Atrens, 1999).

The in vitro degradation rate of $M g$-based implants is commonly assessed by a hydrogen evolution method. The material is immersed in an $\mathrm{NaCl}$ solution, simulated body fluid (SBF) or a balanced salt solution (e.g. Hank's solution), and the amount of dissolved material is measured over a period of a few hours to a few weeks by measuring the amount of hydrogen produced. For an excellent review of other corrosion rate measurement techniques, see (Kirkland et al. 2012).

Since these tests take a substantial amount of time, it would therefore be beneficial to have a mathematical model to estimate the degradation rate of a sample of given geometry. A number of such models are already present in the literature, each of them focusing on a different aspect of the corrosion of $\mathrm{Mg}$. Models based on the Continuum Damage (CD) theory were presented in (Gastaldi et al. 2011) and (Grogan et al., 2011). CD theory, in this case used in a Finite Element (FE) framework, allows the modelling of corrosion resulting from different mechanisms. In (Gastaldi et al. 2011), uniform microgalvanic corrosion is coupled with stress cracking corrosion, whilst the study in (Grogan et al. (2011) focuses on non-uniform, pitting microgalvanic corrosion and ignores the stress cracking. Both models share a purely phenomenological approach to microgalvanic corrosion and are used to predict the loss of ability to maintain load by metallic stents.
A different approach has been proposed in (Grogan et al. 2014), in which the sample degradation is considered as a moving-boundary problem and transport-driven corrosion is modelled. The model focuses on the first hour of corrosion after immersion and hence it ignores the formation of the protective film completely. Partial differential equation models which take into account galvanic corrosion include (Wilder et al., 2015), where the finite differences method coupled with the level-set method is used to simulate the anode-cathode interactions. A very detailed and well-parametrised approach to galvanic corrosion is described in (Sun et al. 2013), where the effects of the corrosion deposits on the degradation are considered.

A semi-quantitative mathematical model based on transport-driven corrosion is proposed in this study which aims to provide a suitable computational framework for the asessment of the degradation rate of the implants prior to the conduction of any in vitro or in vivo experiments.

\section{Computational framework}

\subsection{Corrosion mechanism}

The corrosion of magnesium is a relatively well-studied electrochemical process and can be summarised by the following reactions (Witte et al., 2008):

$$
\begin{aligned}
\mathrm{Mg} & \longrightarrow \mathrm{Mg}^{2+}+2 e^{-}, \\
2 \mathrm{H}_{2} \mathrm{O}+2 e^{-} & \longrightarrow \mathrm{H}_{2}+2 \mathrm{OH}^{-}, \\
\mathrm{Mg}^{2+}+2 \mathrm{OH}^{-} & \longrightarrow \mathrm{Mg}(\mathrm{OH})_{2} .
\end{aligned}
$$

In the anodic reaction in (1) $M g$ ionises to $M g^{2+}$. The electrons then react with water molecules as shown in (2). A fraction of $\mathrm{Mg}^{2+}$ ions reacts with $\mathrm{OH}^{-}$ions to form a protective layer of magnesium hydroxide $\left(\mathrm{Mg}(\mathrm{OH})_{2}\right)$ in the precipitation reaction in (3), whilst the rest dissolves into the surrounding medium. However, in the presence of chloride ions in a salt solution, magnesium hydroxide is converted into magnesium chloride $\left(\mathrm{MgCl}_{2}\right)$ through the following reaction:

$\mathrm{Mg}(\mathrm{OH})_{2}+\mathrm{Cl}^{-} \longrightarrow \mathrm{MgCl}_{2}+2 \mathrm{OH}^{-}$.

$\mathrm{MgCl}_{2}$ has a much higher solubility in water than $\mathrm{Mg}(\mathrm{OH})_{2}$, therefore $\mathrm{Cl}^{-}$ions have the effect of breaking down the protective film and enhancing the corrosion rate (Birbilis et al. 2009). Crucially, one mole of $M g$ dissolved corresponds to one mole of $H_{2}$ produced and hence measuring the weight loss of $M g$ is equivalent to measuring the amount of hydrogen produced. 


\subsection{Level-set method}

The level-set method (Chen et al., 1997, Guyot et al., 2014; Wilder et al. 2015) was chosen in order to capture the complicated and volatile topology of the porous scaffold during its degradation. The domain is subdivided into two timedependent subdomains: $\Omega_{1}$ (the solid) and $\Omega_{2}$ (the medium). The level-set function $\phi$ is a signed distance from the interface with $\phi>0$ in $\Omega_{1}$ and $\phi<0$ in $\Omega_{2}$. The interface between the two domains is denoted by $\Gamma$ and is implicitly given by $\phi=0$. The role of $\phi$ is to implicitly track the movement of the interface $\Gamma$.

If the interface $\Gamma$ moves at a velocity $\mathbf{v}$ (extended continuously to the whole domain), the corresponding level-set equation is

$$
\frac{\partial \phi}{\partial t}+\mathbf{v} \cdot \nabla \phi=0
$$

with a zero-flux boundary condition. This equation ensures, that the interface $\Gamma$ moves at the correct speed.

It is assumed that the movement is in the direction normal to the interface, so that

$\mathbf{v}=v \frac{\nabla \phi}{|\nabla \phi|}$

and equation 5 becomes

$$
\frac{\partial \phi}{\partial t}+v|\nabla \phi|=0
$$

One of the problems associated with equation $(6)$ is that after one iteration of the implicit Euler algorithm used in the simulations, the function $\phi$ ceases to be a signed distance function. To overcome this issue, the level-set function $\phi$ is reinitialised at each time step. The signed distance is calculated explicitly using the open-source software mshdist (Dapgony and Frey, 2012).

\subsection{Equations and variables}

The model takes the form of a set of three reaction-diffusion differential equations and a level-set equation. These are used to describe the time evolution of the three most significant variables participating in the degradation process: the concentrations of magnesium ions, the protective film and the chloride ions, denoted respectively $[\mathrm{Mg}], \mathrm{F}$ and $[\mathrm{Cl}]$, and all of which are measured in $\mathrm{g} / \mathrm{mm}^{3}$.

$F$ is the concentration of the protective film which forms on the surface of the scaffold in reactions (3) and (4). In water it consists only of $\mathrm{Mg}(\mathrm{OH})_{2}$, but in the presence of other ions in the medium it may also contain $\mathrm{MgCl}_{2}\left(\mathrm{Cl}^{-}\right.$ions), $\mathrm{Mg}_{3}\left(\mathrm{PO}_{4}\right)_{2}\left(\mathrm{HPO}_{4}^{2-}\right.$ ions $)$ and giorgiosite ( $\mathrm{HCO}_{3}^{-}$ions).
$\mathrm{Mg}_{3}\left(\mathrm{PO}_{4}\right)_{2}$ and giorgiosite are stable and offer good protection from corrosion. Some authors also report $\mathrm{MgO}$ being present in the protective film (Xin et al., 2011; Song and Atrens, 1999). Here it is assumed that the film consists only of $\mathrm{Mg}(\mathrm{OH})_{2}$.

The model simplifies reactions (1)-(4). Due to the presence of the buffering system in human blood and the experiment, the $\mathrm{pH}$ is assumed to be constant (although there are local changes in $\mathrm{pH}$ on the surface of the implant (Song and Atrens, 2003).

The model takes the following form (note that the equations below are limited to the medium subdomain $\Omega_{2}$ ):

$$
\begin{aligned}
\frac{\partial[M g]}{\partial t} & =\nabla\left(D_{M g}^{e} \nabla[M g]\right)-k_{1}[M g]\left(1-\frac{F}{F_{\max }}\right)+k_{2} F[C l]^{2} \\
\frac{\partial F}{\partial t} & =k_{1}[M g]\left(1-\frac{F}{F_{\max }}\right)-k_{2} F[C l]^{2} \\
\frac{\partial[C l]}{\partial t} & =\nabla\left(D_{C l}^{e} \nabla[C l]\right)
\end{aligned}
$$

where $k_{1}$ and $k_{2}$ are the reaction rates of the film formation (1-3) and film dissolution (4) respectively, $D_{c}^{e}$ denotes the effective diffusion coefficient of a specimen $c$ and $F_{\text {max }}$ is the maximum film concentration. In order to represent the closed environment of the in vitro tests, equations (7t9) were given zero-flux boundary conditions. The whole set-up is shown schematically in Fig. 1 .

The term $k_{1}[M g]\left(1-\frac{F}{F_{\max }}\right)$ responsible for the formation of the protective film is feasible only if $F(\mathbf{x}, t) \leq F_{\max }$. However, for any $\mathbf{x}$ inside the domain, if $F(\mathbf{x}, t)=F_{\text {max }}$, then $\frac{\partial F}{\partial t}(\mathbf{x}, t)=-k_{2} F(\mathbf{x}, t)([C l](\mathbf{x}, t))^{2} \leq 0$. It therefore follows that if the initial conditions are such that $F(\mathbf{x}, 0) \leq F_{\max }$ for all $\mathbf{x}$, then Equations 77 and 8 remain valid for all subsequent times $t$.

The initial conditions were chosen to reflect the experimental setup from (Abidin et al., 2013). Hence $[M g]$ was initially chosen to be $M g_{\text {sat }}$ in the sample and $0 \mathrm{~g} / \mathrm{mm}^{3}$ in the medium. The initial condition for $[\mathrm{Cl}]$ was $0 \mathrm{~g} / \mathrm{mm}^{3}$ in the sample and $4.852 \times 10^{-5} \mathrm{~g} / \mathrm{mm}^{3}$ in the medium. $F$ is taken to be 0 initially since no protective film is present on the surface of the scaffold.

The effective diffusion coefficient is calculated using the theory of diffusion in the porous medium. The $\mathrm{Mg}(\mathrm{OH})_{2}$ film forming on the implant surface is treated as a porous material. In the absence of the film, a specimen $c$ diffuses with a diffusion coefficient $D_{c}$. If the film is fully saturated $\left(F=F_{\text {max }}\right)$, the effective diffusion coefficient is $\frac{\varepsilon}{\tau} D_{c}$ (Grathwohl, 1998, where $\varepsilon$ is the porosity and $\tau$ is the tortuosity of the deposits. Linear interpolation between these two values leads to the following expression for the effective diffusion coefficient:

$D_{c}^{e}=D_{c}\left(\left(1-\frac{F}{F_{\max }}\right)+\frac{F}{F_{\max }} \frac{\varepsilon}{\tau}\right)$. 
It is assumed that the corrosion process is diffusiondriven, which is justified in the case of HP magnesium as the lack of impurities ensures that ion transfer is minimal and that electrochemical corrosion is negligible. The mass transport through the interface leads to the following condition for the shrinkage velocity (Grogan et al., 2014):

$D_{M g}^{e} \nabla_{\mathbf{n}}[M g]-\left(M g_{\text {sol }}-M g_{s a t}\right) v=0$,

where $M g_{s o l}$ is the concentration of magnesium in the implant, $M g_{\text {sat }}$ is the concentration of magnesium at which the solution saturates and $\nabla_{\mathbf{n}}$ denotes the directional derivative in the direction $\mathbf{n}=-\nabla \phi$, i.e. along the outward pointing normal to the interface.

Using the above relation, the level-set equation becomes

$$
\frac{\partial \phi}{\partial t}-\frac{D_{M g}^{e} \nabla_{\mathbf{n}}[M g]}{M g_{s o l}-M g_{s a t}}|\nabla \phi|=0
$$

\subsection{Implementation of the model}

The model was implemented using FreeFem++ software (Hecht, 2012) and the finite element (FE) method was used to numerically solve the system consisting of equations (6) and (7) - (9), a method previously used in (Guyot et al., 2014; Wilder et al., 2015) in a similar context. ParaView was used to visualise the results.

The simulations were conducted using two different two-dimensional geometries: (a) a solid $M g$ block used in the fitting procedure (Fig. 3 and (b) a porous scaffold derived from a nanofocus computed tomography scan (nanoCT) (Lietaert et al. 2013) (Fig. 4).

The finite elements used were triangular and the functions were approximated using first-order polynomials. An adaptive grid was used since it produces stable results over a larger range of parameters than a regular one. This is to be expected as the key processes for degradation happen at the scaffold-medium boundary and are hence strongly localised. The large size of the domain is necessary in order to ensure that the solution does not saturate too quickly with $M g$ as this would stop the degradation process. The built-in FreeFem++ mesh adaptation routine was used.

The numbers of finite elements for (a) and (b) were 238072 and 839542 respectively. Such a high accuracy was needed due to the low degradation rate of high purity (HP) $M g$, which also resulted in no remeshing being needed.

The timestep was taken to be 0.0125 day. Further decreasing the timestep in a convergence analysis had no major influence on the outcome of the simulations.

It should be noted that some difficulties arose with the calculation of the shrinkage velocity $v$ at the interface. To ensure the correct boundary condition at the interface, the numerical scheme sets the value of $[M g]$ in the whole $\Omega_{+}$ subdomain associated with the sample (the exact concentration of $[M g]$ inside the scaffold is irrelevant for the simulations). This is done by penalising the bilinear form associated with the variational formulation of Equation (7) inside $\Omega_{+}$. This allows for non-conformity of the interface with the mesh (as shown in Fig. 2). The fact that the interface cuts through a finite element led to an overestimate of the value of $[M g]$ at the nodes which lay just outside the $\Omega_{+}$ subdomain. To calculate the shrinkage velocity at a point on the interface (Equation $(11)$ ), the values of $\nabla_{\mathbf{n}}[M g]$ and $D_{M g}^{e}$ at a distance $h$ along the outward pointing normal from the interface are used, where $h$ is the radius of the smallest element in the mesh. This procedure is shown schematically in Fig. 2.

The fact that the diffusion only happens outside the magnesium block results in a jump in the diffusion coefficient at the interface. Such jumps are likely to produce local oscillations when numerical schemes are used. In order to overcome this issue, a built-in FreeFem++ mass lumping option qf1pTlump was used when computing the bilinear form associated with Equations (7) and (9). No oscillations were present when this option was invoked.

\subsection{Parameters}

The values of the parameters used are summarised in Table 1.

Data on the reaction rates $k_{1}$ and $k_{2}$ is, to the best of the authors' knowledge, non-existent in the literature. The values of diffusion coefficients used by various authors often differ by as much as an order of magnitude. For example, compare the values $0.2538-12.138 \frac{\mathrm{mm}^{2}}{d a y}$ from (Grogan et al. 2014) with 60.912 $\frac{\mathrm{mm}^{2}}{d a y}$ from (Sun et al. 2013) for the diffusion coefficient of $M g$. These tour parameters were therefore subject to a fitting procedure.

Data from (Abidin et al. 2013) was used in the calibration. This particular data set was chosen for the following reasons: (i) the use of high purity magnesium ensures that corrosion is mostly transport-based, and (ii) $\mathrm{CO}_{2}$ bubbling is used to maintain constant $\mathrm{pH}$. The exact experimental setup is given in (Abidin et al. 2013) and the fitting is discussed in more detail in Section 3

The values of the porosity and tortuosity of the $\mathrm{Mg}(\mathrm{OH})_{2}$ protective film were reported in (Sun et al. 2013). The value of $M g_{\text {solid }}$ is the density of $M g$. Both $M g_{\text {solid }}$ and the saturation concentration $M g_{\text {sat }}$ are obtained from (Grogan et al. 2014). The value of the maximum protective film concentration is taken to be the density of $\mathrm{Mg}(\mathrm{OH})_{2}$ multiplied by the fraction of volume it occupies when treated as a porous material, namely $1-\varepsilon$. 
Table 1 Parameters for the model. The parameters marked with an asterisk were subject to a fitting procedure and the ranges within which they were varied are shown in the Value column.

\begin{tabular}{|c|c|c|c|}
\hline Parameter & Unit & Value & Reference \\
\hline${ }^{*} k_{1}$ & $\frac{1}{d a y}$ & $0-10$ & \\
\hline${ }^{*} k_{2}$ & $\frac{m m^{6}}{\text { day } g^{2}}$ & $10^{4}-10^{12}$ & \\
\hline${ }^{*} D_{M g}$ & $\frac{m m^{2}}{d a y}$ & $10^{-5}-1$ & \\
\hline${ }^{*} D_{C l}$ & $\frac{m m^{2}}{d a y}$ & $10^{-5}-1$ & \\
\hline$\varepsilon$ & - & 0.55 & (Sun et al. 2013) \\
\hline$\tau$ & - & 1 & (Sun et al. 2013) \\
\hline$M g_{\text {solid }}$ & $\frac{g}{m m^{3}}$ & $1.73510^{-3}$ & (Grogan et al. 2014) \\
\hline$M g_{\text {sat }}$ & $\frac{m m^{2}}{m m^{3}}$ & $1.3410^{-4}$ & (Grogan et al. 2014, \\
\hline$F_{\max }$ & $\frac{m}{m m^{3}}$ & $1.2895310^{-3}$ & \\
\hline
\end{tabular}

\subsection{Measurement of the corrosion rate}

The corrosion rate is measured by means of hydrogen formation (or equivalently the weight loss). It can be seen from reactions (1) and (2) that one mole of magnesium dissolved corresponds to one mole of hydrogen formed. It is therefore common experimental practice to capture this gas and assess the corrosion rate by measuring the amount of hydrogen formed. An obvious advantage of such an approach is the ability to continuously measure the corrosion rate without removing the species from the fluid (Xin et al. 2008). It has however been reported that this slightly underestimates the corrosion rate due to the fact that some of the hydrogen may diffuse into the magnesium (Atrens and Winzer, 2006) and this is not taken into the account in our model.

In the model, the amount of dissolved magnesium is measured as follows: the initial amount of magnesium is

$M g_{0}=\int_{\Omega_{+}(0)} M g_{\text {solid }} d V$

where $\Omega_{+}(t)=\{\mathbf{x}: \phi(\mathbf{x}, t) \geq 0\}$. Therefore the total mass loss at time $t$ is:

$M g_{t}=\int_{\Omega_{+}(t)} M g_{\text {solid }} d V-M g_{0}$.

The amount of hydrogen evolution $H_{t}$ per square centimetre of exposed area, which is a value often measured during experiments, is then calculated using the Ideal Gas Law:

$H_{t}=\frac{M g_{t}}{M g_{m o l}} \frac{R T}{P A}$,

where $M g_{\text {mol }}$ is a molar mass of $M g\left(24.305 \frac{g}{m o l}\right), R$ is the universal gas constant, $T$ is absolute temperature ( $310.15 \mathrm{~K}), P$ is the pressure $(1 \mathrm{~atm})$ and $A$ is the initial exposed area.

\subsection{Objectives}

The objective of this study was to develop a computational framework which enables the modelling of the degradation of $M g$. The validity of the model was assessed by conducting simulations using two types of geometry. A solid $M g$ block (Fig. 3) was used in order to calibrate the parameters against the experimental results obtained in (Abidin et al. 2013). A porous $M g$ scaffold (Fig. 4) was used to assess the model's performance in the more practical context of orthopaedic bioimplants.

The model's emphasis is put on the process of formation/dissolution of the protective film, as it is a crucial process in the degradation of $\mathrm{Mg}$. The role of $\mathrm{Cl}^{-}$ions in the dissolution of the protective film is taken into account, as is the effect of the film on the diffusion. The model also follows the release of $M g$ into the medium, as this is important for two reasons: (i) the concentration gradient at the interface influences the dissolution rate, and (ii) the effect of $M g^{2+}$ ions on the bone tissue growth inside the scaffold may be investigated in the future.

The level-set method was used in order to be able to keep track of very complicated geometries, which is crucial when porous orthopaedic implants are involved.

\section{Results}

Four parameters (the reaction rates $k_{1}$ and $k_{2}$ and the diffusion coefficients $D_{M g}$ and $D_{C l}$ ) were varied within the ranges indicated in Table 1. Given the spread of data in Abidin et al. 2013), two calibrations were conducted using two separate data sets $\mathrm{A}$ and $\mathrm{B}$ in order to validate that the model is well-behaved over a large range of parameters. The geometry used in the fitting procedure (which represents the cross-section of the actual sample) is shown in Fig. 3.

The least squares method was used in the fittings. Good fits were obtained with the results shown in Fig. 5(the mean square distance errors were (A) $A=0.000955232$ and (B) $0.028518)$. The exact values of the parameters obtained are as follows: (A) $k_{1}=2, k_{2}=1 \times 10^{9}, D_{M g}=0.00075, D_{C l}=$ 0.24 and (B) $k_{1}=0.3, k_{2}=5.5 \times 10^{1} 0, D_{M g}=0.016, D_{C l}=$ 0.24 . 
The model's aim is to replicate the formation of the protective film on the surface of the sample. The concentration of the protective film at different times after immersion are shown in Fig. 6 for the simple geometry.

Since porous $M g$ scaffold are of interest with regard to orthopaedic applications, simulations were also conducted for a cross-section of a porous HP $M g$ scaffold. The relevant geometry is shown in Fig. 4 A part of the whole computational domain after $0,2,7$ and 35 days after immersion is presented in Fig. 7 to illustrate the degradation and formation of the protective film as predicted by the model. Due to the extremely slow degradation of the HP $M g$, full degradation is not present even a year after immersion (result not shown).

Changes in the geometry of the porous scaffold predicted by the model are shown in Fig. 7. The emphasis is on the changes in topology, as these changes may severely compromise the ability of an implant to maintain load.

Fig. 7 also shows the concentration of a protective film on the surface of the porous implant at different times after immersion. It is worth noting that due to the build-up of the corrosion products and saturation of the solution with $M g$, the corrosion in small pores effectively stops.

Fig. 8 shows the hydrogen evolution as predicted by the model for a porous scaffold. It can be seen that the hydrogen evolution rate is slightly faster in the case of a porous implant than in the case of a solid block. The long-term hydrogen evolution is also shown.

\section{Discussion and conclusion}

A semi-quantitative model for the transport-based dissolution of $M g$-based scaffolds in SBF was developed in this study. The corrosion process for $M g$ was modelled using the level-set method and a set of partial differential equations. The aim was to provide an aid for the design and manufacturing of biodegradable implants. Due to the lack of data on the reaction rates $k_{1}$ and $k_{2}$, and the diffusion coefficients, two calibration procedures were conducted using data reported in the literature (Abidin et al., 2013). The model produces reasonable results over a relatively wide range of parameters, as listed in Table 1 . The ability of the model to handle complex geometries in the context of porous implants was then tested. A long-term simulation was conducted using a nano-CT scan of the cross-section of a porous scaffold.

The parameters were successfully adjusted to fit the experimental data for the corrosion of high purity $M g$ from (Abidin et al. 2013). This shows that the model is likely to be a reasonable approximation of the actual processes taking place on the sample-medium boundary. The model correctly simulates the formation of a protective film on the surface of the scaffold, which effectively inhibits the corrosion rate. The film is usually formed within 1-5 days of immersion in SBF after which the corrosion rate is reported to be about 4-10 times lower than it was initially (Hofstetter et al., 2014, Wen et al., 2009; Xin et al., 2008).

The model described in this study significantly differs from other models present in the literature which are based on the CD theory, e.g. (Gastaldi et al., 2011) and (Grogan et al. 2011). A common feature of these models is a phenomenological approach to the transport and galvanic corrosion - none of them attempts to describe the underlying physical processes. CD models mainly focus on the mechanical integrity of the implant and its ability to maintain load. Our study is different in its nature - its aim is not only to make predictions about the degradation rate, but also to provide some insight into the actual chemical processes occurring at the scaffold-medium boundary, such as the precipitation of the corrosion products. The release of ions into the medium is also considered. The ion release is of importance to the biological processes taking place inside the pores of orthopaedic implants, e.g. $M g^{2+}$ ions enhance bone tissue growth (Xin et al. 2011).

One of the advantages of the CD models is that they can easily include corrosion mechanisms from different sources (i.e. transport, micro- and macrogalvanic and stress cracking corrosion). Our model ignores sources of degradation other than transport-based ones, which limits its applicability. The model may nevertheless in the future replace the phenomenological approach and serve as one of the building blocks for the scalar damage field used in the CD models.

The use of the level-set method allows the tracking of very complex geometries, which makes the model easily applicable to porous orthopaedic implants. The model shows little difference in the degradation rate between the porous and solid geometries, and a possible direct application of the model includes an investigation of the degradation rate as a function of pore size. It is likely that a stagnant solution inside very small pores may saturate quickly with $M g$ ions or become filled with the corrosion products. This may effectively stop the degradation inside the pores and decrease the overall corrosion rate.

Only two-dimensional simulations were performed in this study. In line with (Nava et al., 2013), we therefore expect the parameter values listed in Table 1 to be different for a three-dimensional model and hence another calibration would be required if the model were applied to a threedimensional mesh.

More experimental work is needed in order to estimate the reaction rates $k_{1}$ and $k_{2}$. An experiment which could be used to obtain their values would measure the corrosion rate of HP $\mathrm{Mg}$ in a buffered solution with different $\mathrm{Cl}^{-}$ion concentrations. The degradation rate of the sample and the concentration of $\mathrm{Mg}^{2+}$ ions in the medium could then be mea- 
sured. Based on this, the amount of $M g$ used to form the protective film could be estimated, which would then lead to estimates of the reaction rates.

The fitting used in vitro experimental data and the influence of the organic components present in vivo was not taken into account. It is hypothesised, for example, that proteins and amino-acids form another thin layer on the implant's surface which affects the corrosion process (the exact response depends on the material). For a review of other issues regarding the in vivo-in vitro gap, see (Sanchez et al. 2015).

As mentioned in Section 2.5, data on the diffusion coefficients in the literature is scarce. The value of $D_{M g}$ plays a key role in the degradation rate. The verification of the assumption that in the case of HP $M g$ the degradation is mainly transport-driven would require an experiment to measure the values of $D_{M g}$ in SBF. Experimentally determined values of the diffusion coefficients would also increase the ability of the model to make more accurate quantitative predictions.

It is important to note that the diffusion coefficient $D_{M g}$ of $M g$ obtained in each of the calibrations shown in Fig. 5 differs by 3-4 orders of magnitude from the values reported in the literature. The values used by other authors, however, are typically based on experiments in salt water rather than SBF. SBF usually contains other ions in addition to $\mathrm{Na}$ and $C l$, which may explain this difference.

The rates of the reactions involved in the corrosion process are not available in the literature, hence the ability of the model to predict the corrosion rate is limited. The quantitative results should therefore be treated with caution.

In terms of possible improvement, it is worth noting that the model assumes a constant $\mathrm{pH}$, which is partially justified by the presence of $\mathrm{pH}$ buffers in blood and often in SBF. In the experimental data used in this study, the value of the $\mathrm{pH}$ was maintained constant. Nevertheless, it is reported in (Song and Atrens, 2003) that the local $\mathrm{pH}$ changes on the sample surface favour the formation of the $\mathrm{Mg}(\mathrm{OH})_{2}$ protective film. The next step in the development of the model may therefore involve including the effects of these $\mathrm{pH}$ changes on the degradation rate. This would probably involve the introduction of an additional equation for $\mathrm{pH}$, which would not only increase the computational complexity, but would also increase the number of parameters in the model. The terms involved in the equation for $\mathrm{pH}$ would also be likely to be experiment-specific, as the methods used to maintain the $\mathrm{pH}$ vary between different experimental setups.

To the best of the authors' knowledge, the model presented in this study is one of the first attempts to model the transport-based degradation of $M g$ which emphasises the effects of formation/dissolution of the protective film. No dedicated experiments were performed to estimate the values of the parameters, hence this study is to be treated as of a qualitative nature.
The model offers a basic framework for assessing the in vitro degradation rate of $M g$, and may be used to simulate the degradation behaviour of $M g$ implants with complex geometries. In cases when the implant is needed to provide support for the surrounding tissue for only a certain period of time, the model may serve as an aid for the design of scaffolds which possess the required mechanical properties, while also staying under a specified limit of release products. The results obtained in this study allow the conduction of in silico tests prior to the manufacturing phase and also provide a framework for further investigation of the electrochemical processes responsible for the corrosion of $M g$. Overall, this study provides important information on the dynamics of the evolution of the scaffold geometry.

\section{Compliance with Ethical Standards:}

Funding V. Manhas and Y. Guyot are funded by Belgian National Fund for Scientific Research (FNRS) grant FRFC 2.4564.12. The research leading to these results has received funding from the European Research Council under the European Union's Seventh Framework Programme (FP/2007-2013)/ERC Grant Agreement No. 279100. The nano-CT images were generated using the X-ray computed tomography facilities of the Department of Materials Engineering of the KU Leuven, financed by the Hercules Foundation (project AKUL 09/001: Micro-and nano-CT for the hierarchical analysis of materials).

Conflict of Interest The authors declare that they have no conflict of interest. 


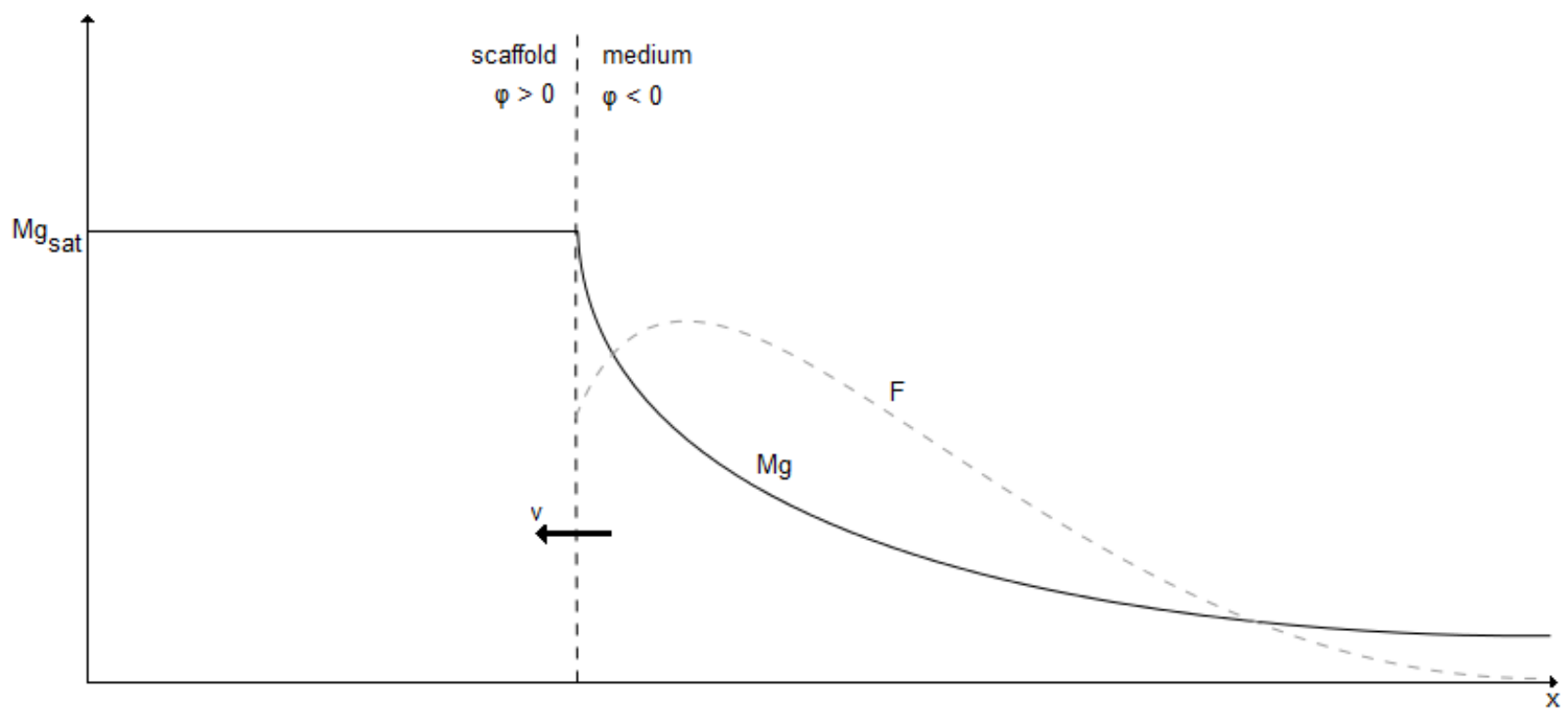

Fig. 1 A one-dimensional schematic diagram of the sample-medium boundary where $\phi$ denotes the level-set function. The concentration of $M g$ (solid black line) inside the scaffold is taken to be $M g_{\text {sat }}$ to ensure the correct boundary condition at the interface. The protective film $F$ (grey dashed line) forms inside the medium and provides a barrier to corrosion by decreasing the diffusion coefficient. The interface moves at a velocity v. $\mathrm{Cl}$ is omitted from the diagram for clarity.

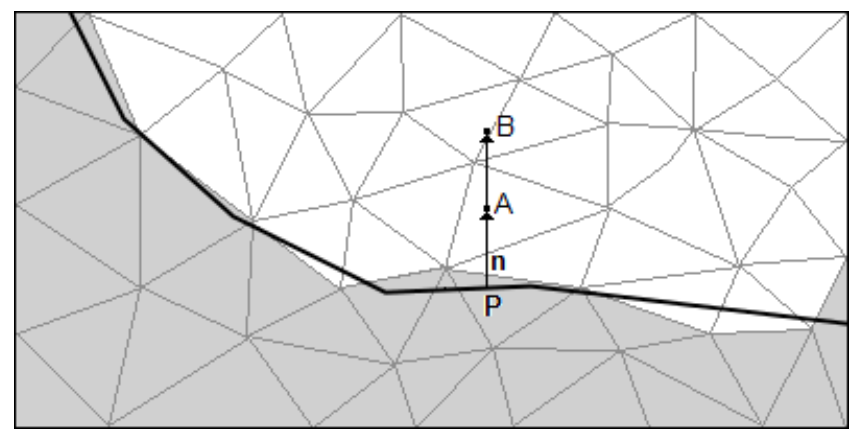

Fig. 2 A schematic figure of the process of calculating the shrinkage velocity. The interface between the solid $M g$ block (grey) and the medium (white) is marked with a solid black line. The implementation of the boundary condition allows for a non-conformity between the interface and the mesh. The symbol $n$ denotes a normal direction at point $P$ on the interface. Points $A$ and $B$ are at a distance $h$ and $2 h$ from the interface respectively and are used in the calculation of $\nabla_{\mathbf{n}}[M g]$. 

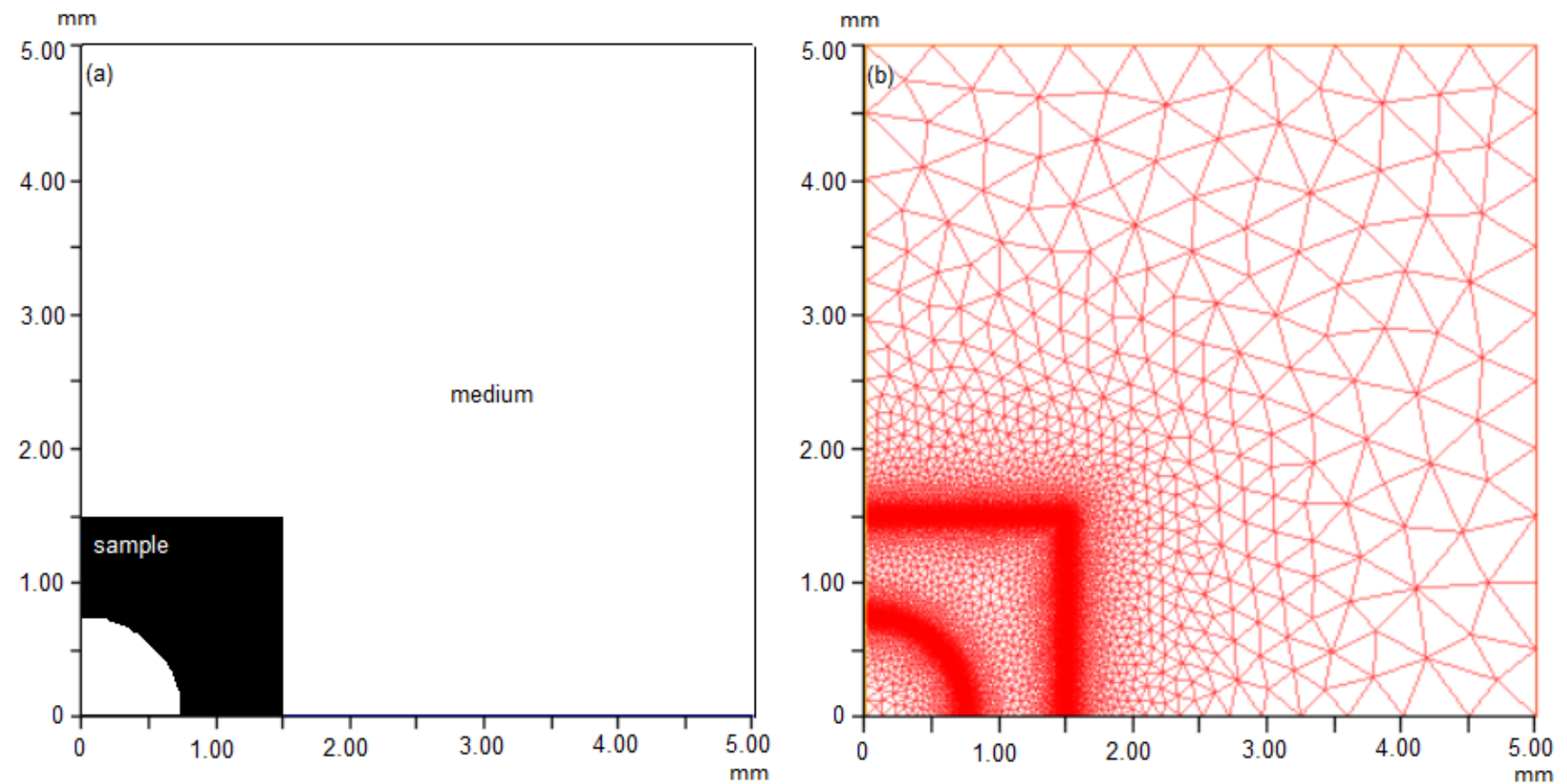

Fig. 3 (a) The geometry of a cross-section of the sample used in the experiments in (Abidin et al. 2013). By symmetry, only a quarter of the cross-section is considered. (b) The associated mesh used in calibration procedure. 


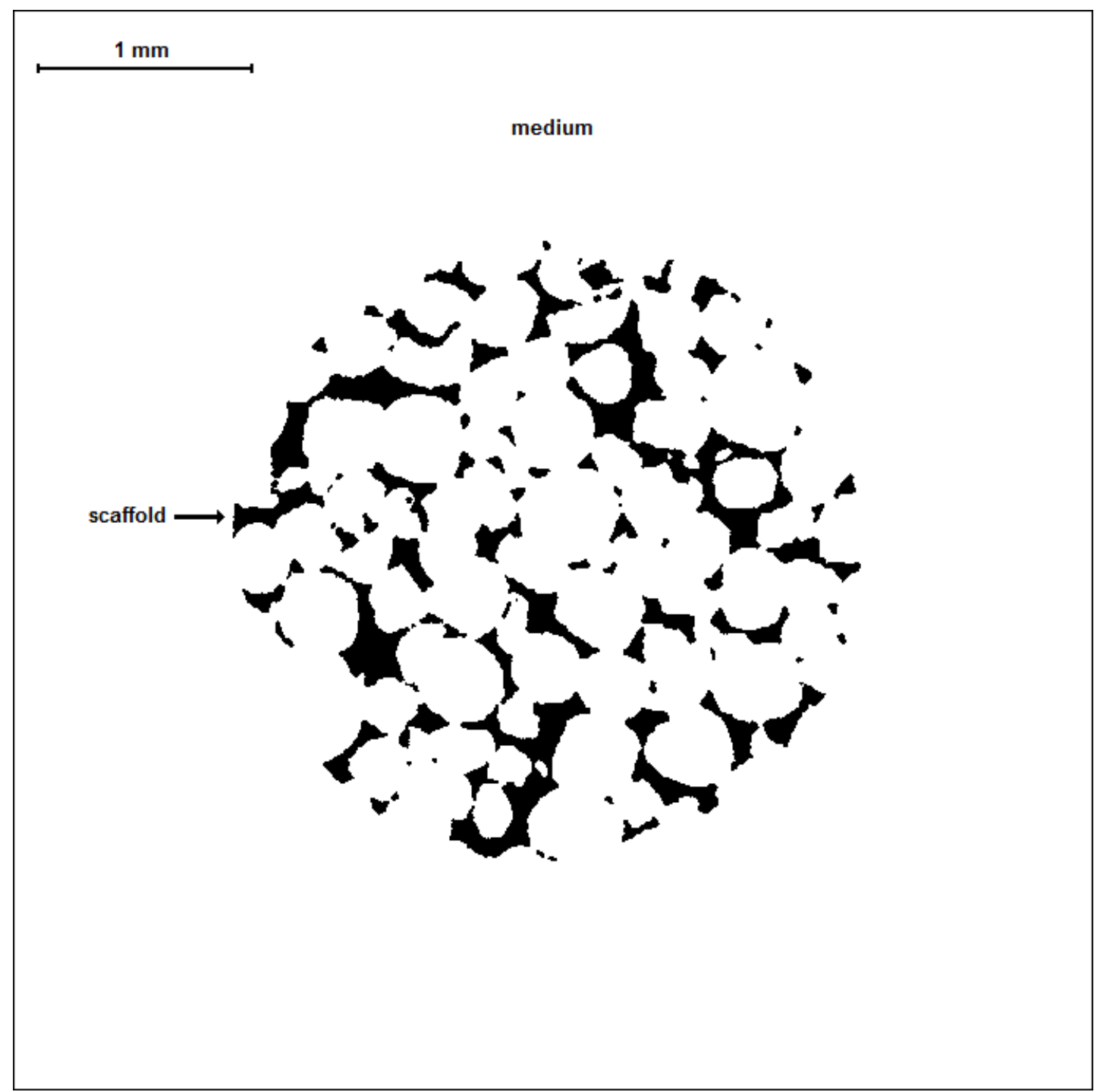

Fig. 4 A porous scaffold (black) geometry used in the simulations. The geometry was obtained from a nano-CT image from (Lietaert et al. 2013). 


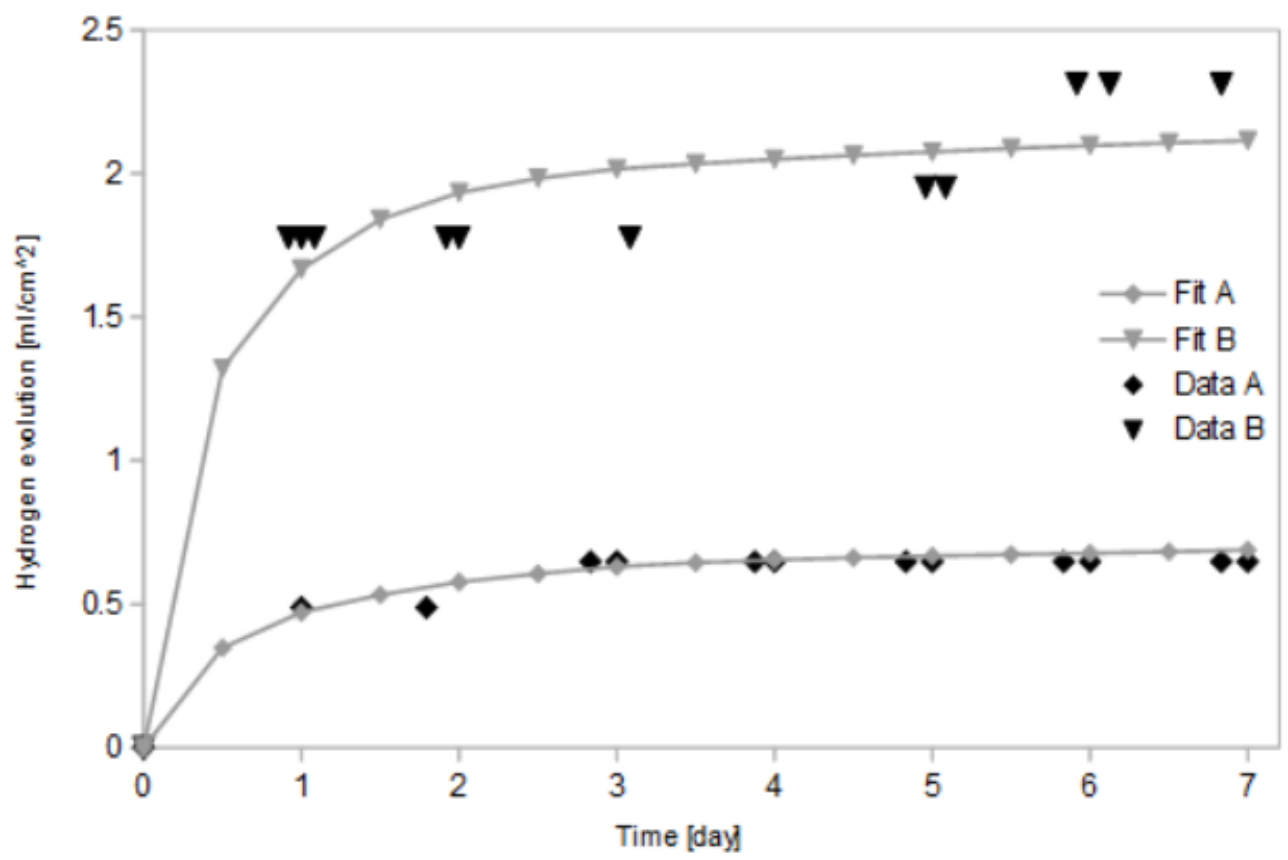

Fig. 5 Hydrogen formation plotted against immersion time. Best fits (grey) to two of the sets of experimental data (black) from (Abidin et al. 2013) are shown.

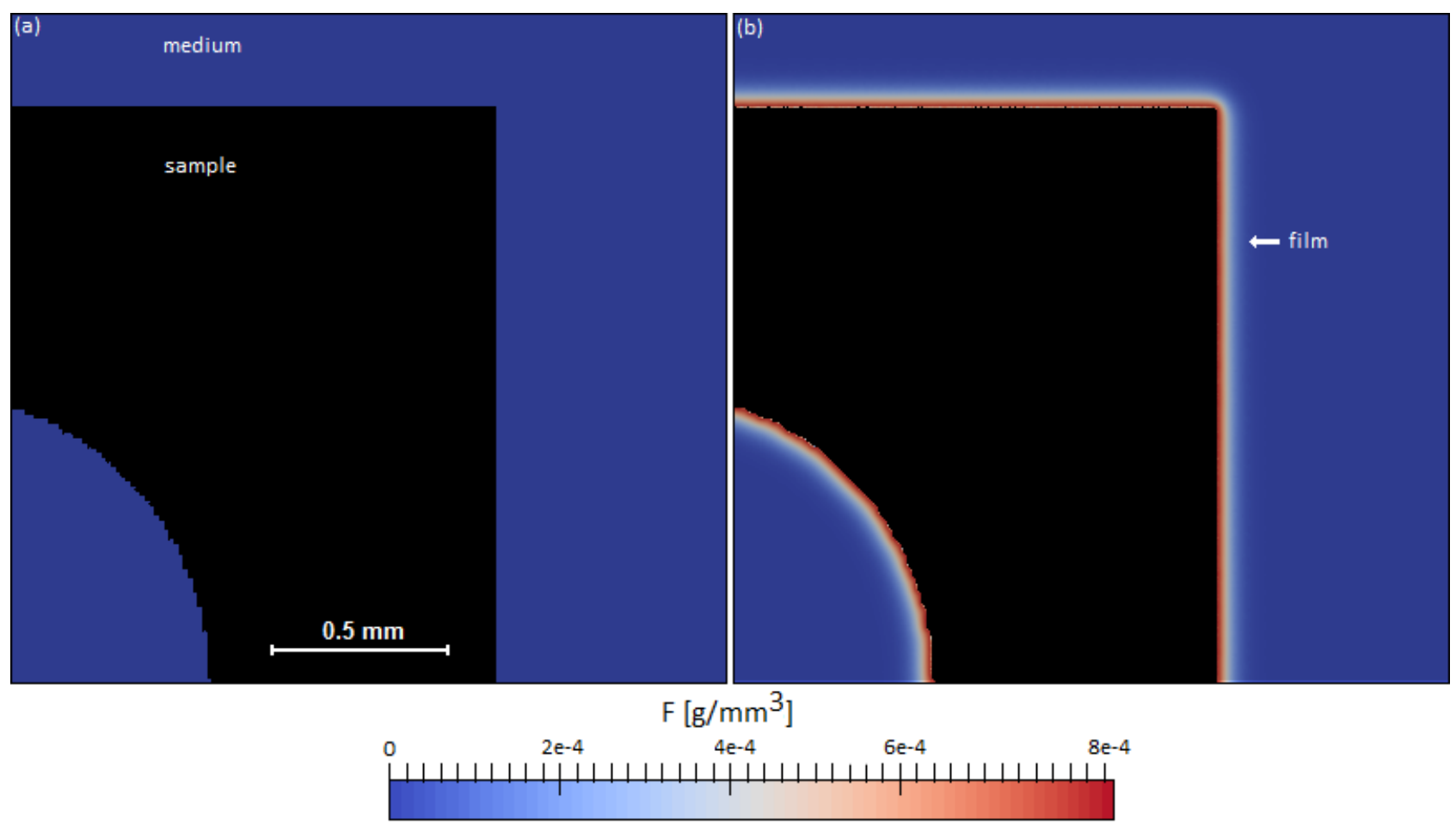

Fig. 6 The protective film formation predicted by the model. Starting with no film at $t=0$ days (a), a thin protective layer (arrow) is visible after $t=7$ days of immersion (b). 

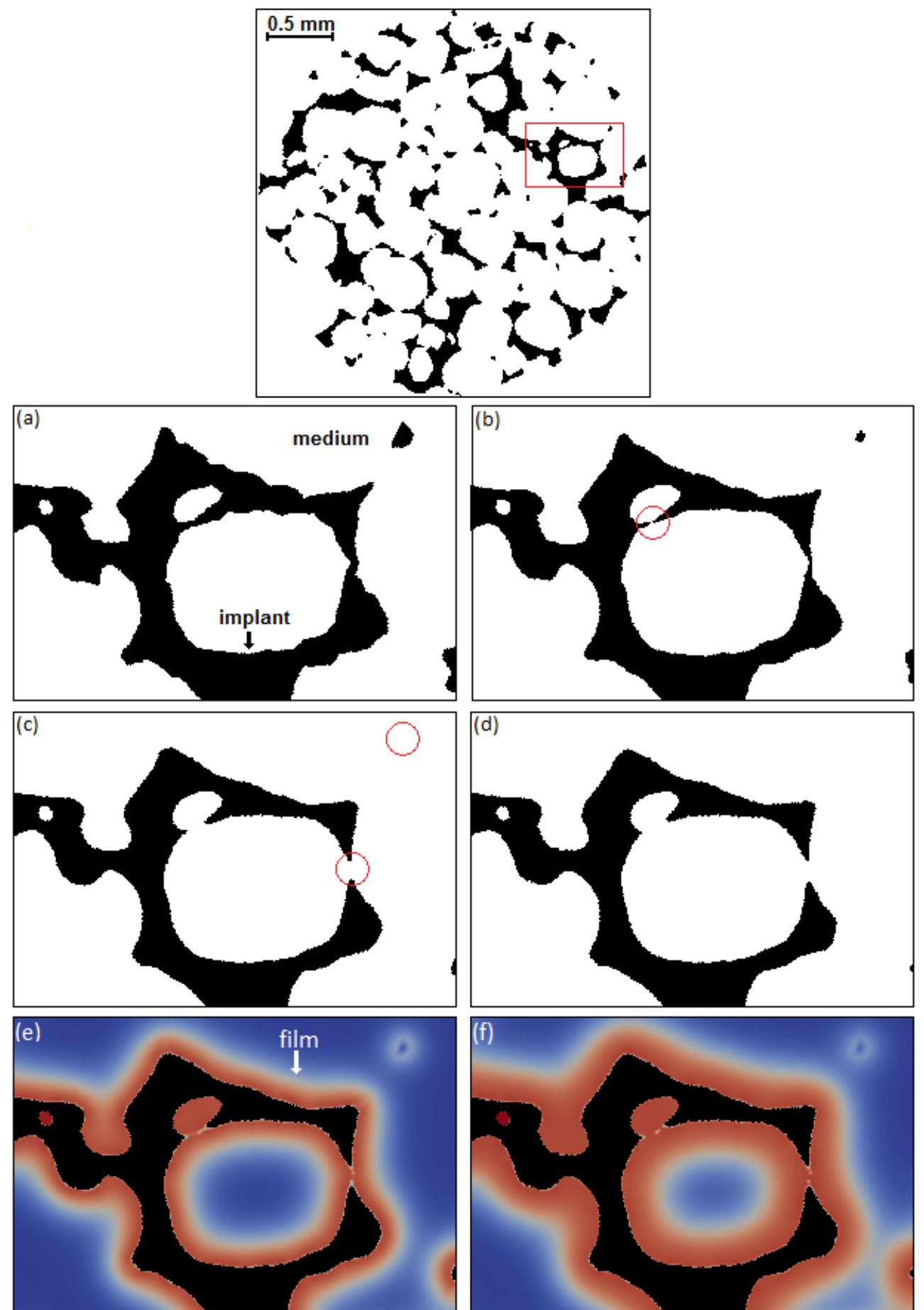

\section{$\mathrm{F}\left[\mathrm{g} / \mathrm{mm}^{3}\right]$}
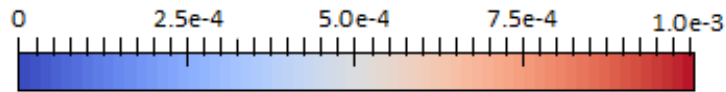

Fig. 7 The changes in topology ((a)-(d)) and the protective film formed ((e), (f)) are shown for a magnified region (red box) of the porous implant as predicted by the simulations. Changes in topology at (a) $t=0$, (b) $t=2$, (c) $t=7$, (d) $t=35$ days of immersion are marked by red circles. Subfigures (e) and (f) show the concentration of the protective film (white arrow) at $t=14$ and $t=28$ days of immersion respectively. 


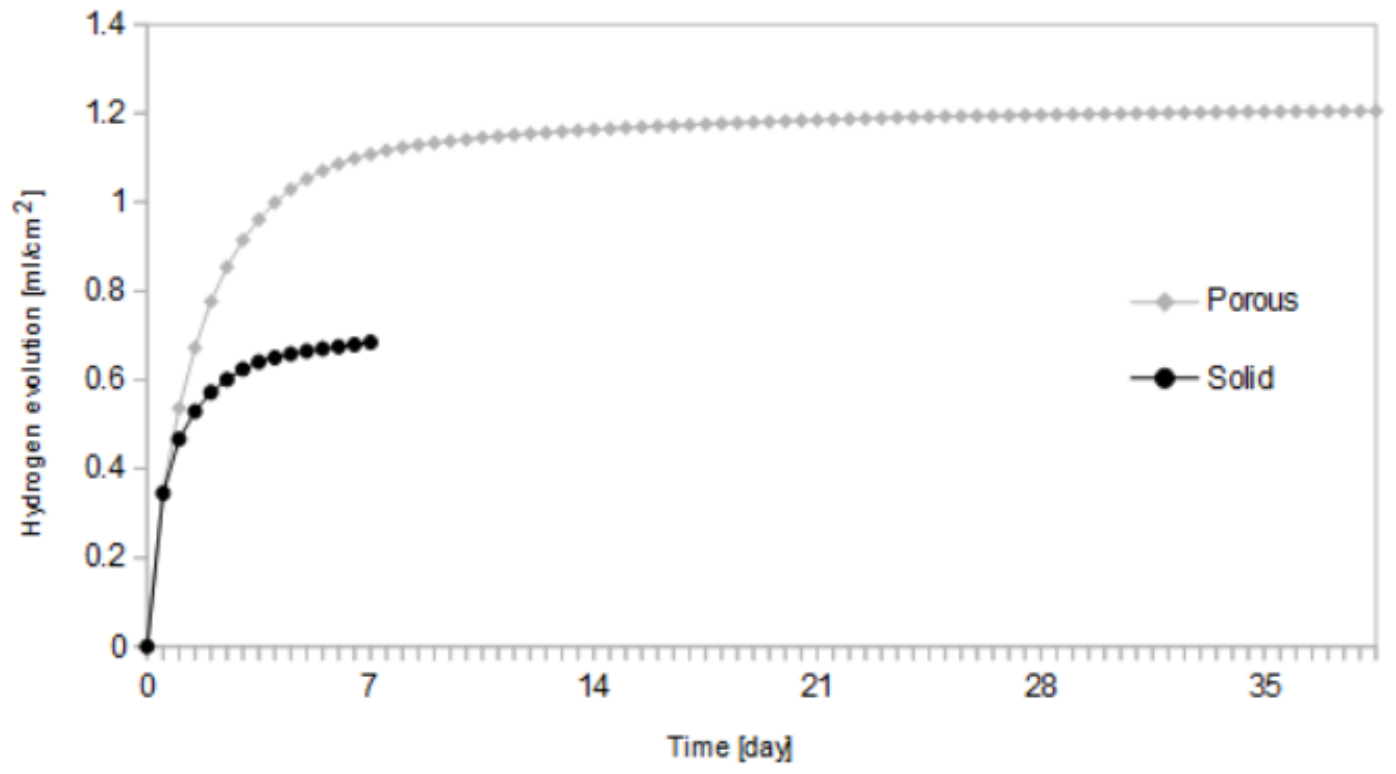

Fig. 8 A graph of the hydrogen evolution for the porous scaffold (grey diamonds) as predicted by the model. The parameter values used are the ones obtained from the fit to the dataset (A). The hydrogen evolution for a solid block (black circles) over the first 7 days is shown for comparison. 


\section{References}

Abidin, N. I. Z., Rolfe, B., Owen, H., Malisano, J., Martin, D., Hofstetter, J., Uggowitzer, P. J., and Atrens, A. (2013). The in vivo and in vitro corrosion of high-purity magnesium and magnesium alloys WZ21 and A91. Corrosion Science, 75:354 - 366 .

Atrens, A. and Winzer, N. (2006). Stress corrosion cracking and hydrogen diffusion in magnesium. Advanced Engineering Materials, 8:749-750.

Birbilis, N., Easton, M. A., Sudholz, A. D., Zhu, S. M., and Gibson, M. A. (2009). On the corrosion of binary magnesium-rare earth alloys. Corrosion Science, 51:683689.

Chen, S., Merriman, B., Osher, S., and Smereka, P. (1997). A simple level set method for solving Stefan problems. Journal of Computational Physics, 135:8-29.

Dapgony, C. and Frey, P. (2012). Computation of the signed distance function to a discrete contour on adapted triangulation. Calcolo, 49:193-219.

Gastaldi, D., Sassi, V., Petrini, L., Vedani, M., Trasatti, S., and Migliavacca, F. (2011). Continuum damage model for bioresorbable magnesium alloy devices - Application to coronary stents. Journal of the Mechanical Behavior of Biomedical Materials, 4:352-365.

Grathwohl, P. (1998). Diffusion in natural porous media: Contaminant transport, sorption / desorption and dissolution kinetics. Springer US.

Grogan, J. A., Leen, S. B., and McHugh, P. E. (2014). A physical corrosion model for bioabsorbable metal stents. Acta Biomaterialia, 10:2313-2322.

Grogan, J. A., O'Brien, B. J., Leen, S. B., and McHugh, P. E. (2011). A corrosion model for bioabsorbable metallic stents. Acta Biomaterialia, 7:3523-3533.

Guyot, Y., Papantoniu, I., Chai, Y. C., Van Bael, S., Schrooten, J., and Geris, L. (2014). A computational model for cell/ECM growth on 3D surfaces using the level set method: a bone tissue engineering case study. Biomechanics and Modeling in Mechanobiology, 13:13611371.

Hecht, F. (2012). New development in FreeFem++. Journal of Numerical Mathematics, 20:251-265.

Hofstetter, J., Martinelli, E., Weinberg, A. M., Becker, M., Mingler, B., Uggowitzer, P. J., and Löffler, J. F. (2014). Assessing the degradation performance of ultrahighpurity magnesium in vitro and in vivo. Corrosion Science, 91:29-36.

Kirkland, N. T., Birbilis, N., and Staiger, M. P. (2012). Assessing the corrosion of biodegradable magnesium implants: A critical review of current methodologies and their limitations. Acta Biomaterialia, 8:925 - 936.

Lietaert, K., L. Weber, J. V., Mortensen, A., Luyten, J., and Schrooten, J. (2013). Open cellular magnesium alloys for biodegradable orthopaedic implants. Journal of Magnesium and Alloys, 1:303-311.

Mueller, W.-D., de Mele, M. F. L., Nascimento, M. L., and Zeddies, M. (2009). Degradation of magnesium and its alloys: Dependence on the composition of the synthetic biological media. Journal of Biomedical Materials Research, 90:487-495.

Nava, M. M., Raimondi, M. T., and Pietrabissa, R. (2013). A multiphysics 3D model of tissue growth under interstitial perfusion in a tissue-engineering bioreactor. Biomechanics and Modeling in Mechanobiology, 12:1169-1179.

Sanchez, A. H. M., Luthringer, B. J. C., Feyerabend, F., and Willumeit, R. (2015). Mg and Mg alloys: How comparable are in vitro and in vivo corrosionrates? A review. Acta Biomaterialia, 13:16-31.

Song, G. (2007). Control of biodegradation of biocompatible magnesium alloys. Corrosion Science, 49:1696-1701.

Song, G. and Atrens, A. (1999). Corrosion mechanisms of magnesium alloys. Advanced Engineering Materials, $1: 11-33$.

Song, G. and Atrens, A. (2003). Understanding magnesium corrosion: A framework for improved alloy performance. Advanced Engineering Materials, 5:837-858.

Staiger, M. P., Pietak, A. M., Huadmai, J., and Dias, G. (2006). Magnesium and its alloys as orthopedic biomaterials: A review. Biomaterials, 27:1728-1734.

Sun, W., Liu, G., Wang, L., Wu, T., and Liu, Y. (2013). An arbitrary Lagrangian-Eulerian model for studying the influences of corrosion product deposition on bimetallic corrosion. Journal of Solid State Electrochemistry, 17:829-840.

Wen, Z., Wu, C., Dai, C., and Yang, F. (2009). Corrosion behaviors of $\mathrm{Mg}$ and its alloys with different $\mathrm{Al}$ contents in a modified simulated body fluid. Journal of Alloys and Compounds, 488:392-399.

Wilder, J. W., Clemons, C., Golovaty, D., Kreider, K. L., Young, G. W., and Lillard, R. S. (2015). An adaptive level set approach for modeling damage due to galvanic corrosion. Journal of Engineering Mathematics, 91:121 - 142.

Witte, F., Hort, N., Vogt, C., Cohen, S., Kainer, K. U., Willumeit, R., and Feyerabend, F. (2008). Degradable biomaterials based on magnesium corrosion. Current Opinion in Solid State and Materials Science, 12:63-72.

Xin, Y., Hu, T., and Chu, P. K. (2011). In vitro studies of biomedical magnesium alloys in a simulated physiological environment: A review. Acta Biomaterialia, 7:14521459.

Xin, Y., Huo, K., Tao, H., Tang, G., and Chu, P. (2008). Influence of aggressive ions on the degradation behavior of biomedical magnesium alloy in physiological environment. Acta Biomaterialia, 4:2008-2015. 